\title{
El proceso de la educación superior en el Perú. La descolonialidad del saber universitario
}

The process of higher education in Peru. The decoloniality of university knowledge

Julio Mejía (jvmena1@hotmail.com) Facultad de Ciencias Sociales, Universidad de San Marcos (Lima, Perú) ORCID: 0000-0002-4170-5643

\begin{abstract}
The paper presents some initial proposals on the development of the university in Peru. The research is divided into four sections. The first one studies the essential historical trends that define the current university. The second, describes the influence of technology and its changes upon college education. The third shows the divergent relations between heterogeneity of knowledge and higher education, mainly monocultural. Finally, the fourth section discusses the possibilities of development in the university.
\end{abstract}

Key words: higher education, university, knowledge, heterogeneity, decoloniality.

\section{Resumen}

El trabajo presenta algunas proposiciones iniciales sobre el desarrollo de la universidad peruana. La investigación se divide en cuatro partes. La primera sección, estudia las tendencias históricas esenciales que definen la universidad actual. La segunda, describe la influencia de los cambios tecnológicos en la educación universitaria. La tercera, muestra las relaciones divergentes entre heterogeneidad de saberes y la educación superior principalmente monocultural. La cuarta parte, discute las posibilidades de desarrollo de la universidad.

Palabras clave: educación superior, saber universitario, heterogeneidad, descolonialidad.

\section{Introducción}

El tema de la universidad peruana no solo ha adquirido importancia en los últimos años, de alguna forma impulsado por la nueva Ley 30220 de 2014, sino que se ha revelado fundamental para comprender la naturaleza de la sociedad peruana.

El artículo pretende analizar las tendencias fundamentales del desarrollo histórico de la universidad peruana que signan su constitución presente. Caminos que establecen una relación de continuidad en el armazón de la universidad. El proceso de la universidad se encuentra relacionado indiscutiblemente a la historia de la Universidad de San Marcos. Destacar el desarrollo de San Marcos es trazar el sugestivo proceso que muestra cómo la universidad peruana está directamente relacionada, en buena parte, con su obra educativa. 
Lo central del análisis consiste en mostrar que la universidad es parte de las relaciones cognoscitivas eurocéntricas, trazadas por la conformación del contenido que asume la modernización en el Perú. Donde modernidad y colonialidad son dimensiones interrelacionadas de una misma realidad. El debate permite situar a la universidad en el horizonte de la colonialidad y, a la vez, proyectarla más allá de los limites definidos por la modernidad.

Por ello, el trabajo presenta para su discusión algunas proposiciones iniciales sobre el desarrollo de la universidad peruana. La investigación se divide en cuatro partes. La primera sección, estudia las tendencias históricas esenciales que definen la universidad actual. La segunda, describe la influencia de los cambios tecnológicos del conocimiento y la comunicación en la educación universitaria. La tercera, muestra las relaciones divergentes entre heterogeneidad de saberes y la educación superior principalmente monocultural. La cuarta parte, discute las posibilidades de los varios desarrollos de la universidad en el Perú.

\section{El proceso de la universidad peruana}

Trazar la marcha histórica de San Marcos permite subrayar el entramado central del proceso de conformación de la universidad peruana. Aquí interesa subrayar las tendencias fundamentales que delimitan el carácter de la universidad realmente existente del siglo XXI.

San Marcos se funda en 1551 como universidad teológica, durante los dos primeros siglos XVI y XVII se organiza en función de prácticas pastorales de la conquista espiritual de las poblaciones indígenas y la dominación del Perú. San Marcos fue creada expresamente como "Real y Pontificia" (Burga 2008:22), enlazaba la presencia del Estado colonial que garantizaba sus rentas principales y el poder de la Iglesia, en particular de los Dominicos, encargado de la labor académica pastoral y del acceso de los docentes. Este tipo de universidad tenía por finalidad "iluminar" con la palabra de Dios a los conquistadores y prepararlos para catequizar a las poblaciones indígenas sometidas con la pretensión de formar hombres cristianos y occidentalizados, según la visión europea y la expansión de la modernidad que siguió a la conquista del nuevo mundo.

A la universidad solo accedían los que consiguieran probar su "sangre limpia" (Loayza 2006:46), de ser parte de las redes familiares de conquistadores y sus descendientes, libres de cualquier mezcla con indios y otras castas inferiores. La Universidad de San Marcos se estructuró en términos de la exclusión racial de las poblaciones indígenas consideradas naturalmente inferiores y, lo más importante, de los saberes nativos destruidos y sometidos por la consideración colonial de la superioridad epistemológica de los conocimientos europeos. Cuando los pocos conocimientos de los pueblos indígenas participan de la universidad, era para ser incorporados a la fe cristiana, aislándolos de su contexto histórico y despojarlos de su identidad. La organización de la universidad de San Marcos se asemejaba al poder panóptico que trazaría la modernidad: el acceso, control y vigilancia cotidiana fueron rigurosamente exigidos a los alumnos y docentes. De esa forma, la Universidad de San Marcos establece un patrón que educa en la catequización para la conquista espiritual, transfigura la vida indígena en "desechable" y los convierte en objetos "excluidos", solo para ser explotados y dominados por las necesidades de la expansión europea.

En ese contexto, no es de extrañar que San Marcos estableciera un orden académico disciplinar y de control por medio de "facultades" y cátedras, que funcionaba en compartimientos estancos cerrados, estableciendo una jerarquía vertical y autoritaria del saber cristiano europeizado. La universidad se apertura con las facultades de Teología y Arte, agregándose a final del virreinato las de Filosofía, Leyes y 
Medicina. La teología ocupará el lugar esencial en la estructura cognoscitiva: el conocimiento es la expresión acabada de la revelación divina de la creación que se orientaba bajo el poder pastoral y evangelizador de la universidad en los primeros siglos de colonia. De esta forma, se sientan los fundamentos de la episteme eurocéntrica que marcan el horizonte de la modernidad, representa el imaginario, la memoria histórica y la perspectiva de conocimiento que permite estructurar todas las subjetividades de los pueblos en un solo gran discurso universal que tienen su eje de poder principal en la Europa del sur, imponiendo una forma de pensamiento que liquidan, subordinan e invisibilizan a los saberes indígenas. La universidad teológica buscaba imponer un pensamiento homogéneo universal y trataba de destruir la heterogeneidad epistémica del nuevo mundo.

Durante los dos primeros siglos la universidad peruana era una forma de colonialidad porque ordenó su contenido epistémico a los medios pastorales de los conquistadores europeos y criollos, pero, sobre todo, condenó a la exclusión la espiritualidad de las poblaciones indígenas y se organizó en el disciplinamiento de los saberes católicos de la inicial modernidad del sur de Europa.

Por otra parte, desde la segunda mitad del siglo XVIII se establecen variaciones significativas en la Universidad de San Marcos que la marcan hasta la primera parte del siglo XX, proceso que se inicia con las reformas de los Borbones y la irradiación de nuevas corrientes del pensamiento de la llustración. El Virrey Manuel de Amat se encargó de organizar la reforma en la educación y la universidad por real orden del 25 de octubre de 1768 y la elaboración de las constituciones para la Real Universidad de 1771. Reformas borbónicas que se dieron como un intento tardío para incrementar el poder del Estado, aminorar la influencia política de la aristocracia criolla y recuperar el poderío español. Al igual que lo ocurrido en otros países de Europa, la dinastía de los Borbones aspiró hacer del Estado el eje principal de la práctica gubernamental.

El Estado asumió el control de la Universidad de San Marcos, su administración, el financiamiento, la estructura académica, los planes de estudio y la selección de los catedráticos. Se pierde la autonomía universitaria en nombre de la "Razón de Estado", ahora convertida en la técnica de gobierno encargada de "ordenar" y "reglamentar" todos los elementos de la sociedad. Pareciera que las propuestas borbónicas intentan un reacomodo en la marcha académica de la universidad. Proponen con poco éxito la creación de facultades técnicas que buscaban la disminución relativa de la importancia de la teología, porque en gran parte se había cumplido con la evangelización de las almas. Sin embargo, en la universidad la vieja herencia y la preminencia aristocrática impulsó más una legitimidad de la Facultad de Artes y Filosofía, propiciando que en la educación superior predomine una combinación de la cultura elitista criolla y del discurso retórico, entre el "sentido aristocrático y un concepto eclesiástico y literario" (Mariátegui 1971: 106).

En efecto, la Universidad de San Marcos seguía manteniendo la estructura disciplinar y arbórea de la organización académica, que tratará de introducir la enseñanza de conocimientos útiles y prácticos. Se crean dos facultades: la de Medicina en 1856 y la de Ciencias Políticas y Administración en 1876, a la par que empiezan a funcionar la Escuela de Puentes, Calzadas y Caminos en 1876. Las nuevas facultades y escuelas intentarían buscar el desarrollo de conocimientos útiles para construir caminos, levantar puentes, mejorar las cosechas, combatir las enfermedades. De esa forma, la ciencia se empieza definir como una forma racional para hacer comprensible el mundo con el propósito de buscar acciones de utilidad social y según los intereses del Estado. Pero la introducción de conocimientos útiles y la revalorización de los "oficios mecánicos" llevan a la necesidad de que los estudiantes adquirieran un conjunto de "competencias" prácticas. Competencias que los aristócratas criollos no estaban dispuestos a aceptar: los 
"oficios mecánicos" eran propios de indios, mestizos y no de la "gente decente" que estudiaba en San Marcos. Las reformas fracasaron, devinieron en un discurso retórico que no podía desplegarse en un contexto dominado por el privilegio de casta. La raíz colonial de la universidad no pudo alterarse, la colonialidad penetraba todo su andamiaje cultural, la función principal era de formar en los hijos de las familias oligárquicas doctores y letrados para los cargos públicos y las órdenes religiosas, negando el acceso de las mayorías indígenas y, sobre todo, de sus conocimientos ancestrales.

En ese sentido, cobra importancia el cultivo retórico de la Filosofía y las Artes en la Universidad de San Marcos dado el interés principalmente de la sociedad aristocrática colonial y el objetivo de la "Razón de Estado" para occidentalizar y aniquilar lo indígena de la vida cultural y social. La moralización de las costumbres y el fomento de los valores del "buen hablar" y del "buen sentir" (Castro-Gómez 2014) recae, sobre todo, en la persistencia de la distinción de estatus de las "gentes decentes" criollas-blancas con las poblaciones indígenas, del ciudadano con el campesino y del civilizado con el bárbaro. Las viejas clases dominantes, para diferenciarse culturalmente y racialmente de los otros sectores sociales, recurrieron a la educación literaria y las humanidades caracterizadas por el discurso retórico y docto que permitían excluir a los sectores indígenas. El culto retórico de las humanidades facilitaba dirigir la universidad hacia la formación de letrados y doctores, diferenciados del trabajo manual y deshonroso de las poblaciones indígenas. La distinción de estatus se sustentaba en el carácter aristocrático que permitía distinguirse no solo culturalmente sino, principalmente, de los cuerpos racializados, mediante hábitos, estilos y comportamientos de casta.

Herencia de la colonialidad en la Universidad de San Marcos que se extiende hasta la primera parte del siglo XX. De la misma forma, con la Independencia se propiciaron ideas de la llustración y la igualdad social que se transformaron en discursos vacíos, carentes de contenidos, donde solo predominaban el viejo espíritu oligárquico-criollo. La mayoría de la población, según el censo de 1876 , comprendía al $57 \%$ de la población indígena que se encontraban impedidas de participar en la Universidad de San Marcos por su condición de inferioridad racial y costumbres bárbaras.

La universidad era el campo de la reproducción de la estructura de colonialidad, el privilegio aristocrático de la educación era la distinción de las casta blanca-criolla y la exclusión indígena ejercida por medio del acceso y control directo de las personas. Al principio de la "sangre limpia" y los saberes y prácticas eclesiásticas del cuidado de las almas de los primeros siglos de la conquista desde el siglo XVIII, se acentuaría el sometimiento de los cuerpos racializados mediante el dominio oligárquico-criollo y retórico de las "buenas costumbres" y prácticas "civilizadas".

Con la crisis del Estado oligárquico y el desarrollo de la globalización se inicia un nuevo proceso de la vida universitaria peruana. Se destaca el Estatuto General de la Universidad Peruana de 1946 y, posteriormente, la Ley Universitaria 13417 de 1960. A partir de la segunda mitad del siglo XX se genera una "reforma silenciosa" cuyo contenido modernizador se proyecta hasta el presente, siendo el Estado uno de los actores protagónicos principales, juntamente con el sector privado, que buscan impulsar la mercantilización de la educación superior.

Modernización de la universidad peruana que tuvo su empuje inicial en la Reforma de Córdoba (1918), que recogía el espíritu demoliberal estudiantil contra los viejos privilegios de casta aristocráticos. La respuesta fue cerrar la Universidad de San Marcos (1932-1935), silenciar su ideario con los regímenes dictatoriales o absorberla por los propios intentos de reforma modernizante gestados desde el Estado. 
Precisamente, la crisis del Estado oligárquico se traduciría en la desarticulación de las relaciones agrarias tradicionales y la desintegración de la arcaica autoridad local y gubernamental, que llevó a la masiva migración de las poblaciones rurales y la profunda urbanización de la sociedad peruana, condicionando la gestación de otro paradigma de universidad peruana. Era evidente que el modelo de universidad colonial había fracasado y se hacía urgente la implementación de nuevas prácticas de gobierno capaces de controlar el desborde y barbarie de las poblaciones migrantes. Empieza a aflorar lentamente la idea de que el objetivo del Estado no consiste en intervenir directamente en el control disciplinar sobre los cuerpos mediante la limpieza de sangre, más bien, se fomenta la idea de que el Estado debe concentrar su acción en la generación de entornos favorables para el desarrollo y en las "condiciones del mercado" para la educación a través de una serie de acciones y políticas públicas.

Como resultado del nuevo escenario histórico, la universidad se masifica y mercantiliza. La centralidad de la Universidad de San Marcos se rompe y desliza hacia un sistema masificado y heterogéneo de educación superior. Hasta 1950 solo existían 5 universidades, 4 estatales y 1 privada, con alrededor 20.000 estudiantes. En 1970 se contaba con 34 universidades, 20 de ellas estatales, y 111.572 estudiantes (Ribeiro 2010:296). Para el año 2017 se cuenta con 143 universidades, 92 son privadas y 51 públicas y aproximadamente 1.125.000 de alumnos. Expansión acelerada de un sistema universitario que incorpora a los nuevos sectores medios y populares de las ciudades del país, generando un sistema educativo muy estratificado y dual, estableciendo una universidad de élite y privada para los sectores más acomodados, mientras que para las colectividades de menores ingresos se cuenta con la universidad masificada pública y de la mayoría de universidades privadas de muy baja calidad. En esas condiciones, el impulso estatal a la modernización de la universidad supone la idea de que la pobreza del país se relaciona con el "subdesarrollo" y la carencia de competencias de los jóvenes. Se propone una educación emprendedora y laboral que permita enfrentar las posibilidades del mercado. Sin embargo, pareciera que la modernización de la universidad peruana traduce el predominio del discurso retórico y claramente expresa las políticas neoliberales que buscan reorientar el viejo privilegio de casta oligárquico de la educación de calidad por un privilegio principalmente mercantil y de riqueza.

Al lado de la masificación del sistema de la universidad, el Estado facilita el desarrollo de un proceso muy pronunciado de mercantilización. La privatización de la universidad peruana ocurre no solo como resultado de un marco jurídico sino, principalmente por la disposición expresa del mismo Estado, para el año de 1980 es esencial el aporte del tesoro público al presupuesto de las dos principales universidades privadas: el 35\% para la Pontifica Universidad Católica del Perú y el 71,7\% de la Universidad Cayetano Heredia (Montoya 2009). Para el 2016, utilizando otros mecanismos de distribución de las finanzas gubernamentales, se sigue favoreciendo a las universidades privadas. De lo presupuestado, el Estado asigna 2.500 millones a las universidades estatales. El Programa Beca 18, con los mismos objetivos de favorecer en forma gratuita a los estudiantes de bajos ingresos, dispone de 927 millones, que representan el $37 \%$ del total percibido por las universidades, sumas inmensas que se dirigen en un $90 \%$ para financiar las instituciones educativas privadas con fines de lucro (Lynch 2016). Hecho muy grave, porque coincide con la disminución del importe estatal a la universidad pública, que como hemos visto se trasfiere directamente al financiamiento nacional a la universidad privada.

De esta forma, la modernización de la universidad peruana conlleva el desarrollo del modelo neoliberal estimulado por el Estado, fundado en la proliferación de universidades de muy baja calidad y en la propagación de profesiones regidas solo por las demandas del mercado. La merma estatal a la universidad pública es parte del proceso de privatización de los servicios de educación, salud y seguridad propiciado por la globalización neoliberal que se desarrolla desde los años 80 . Las políticas neoliberales están 
transformando profundamente la universidad peruana en parte de la valorización del mercado educativo. La expansión de la universidad peruana desde la segunda mitad del siglo XX está llevando a la hegemonía del neoliberalismo en la educación superior. En realidad, las políticas neoliberales denotan la voluntad de desarrollar un mercado universitario donde predominen la privatización e inversión de capital y las preferencias de la riqueza mercantil.

La educación superior expresa la continuidad del patrón de la conquista y de la colonialidad. El Estado peruano, cuando alude a las mayorías poblacionales, "no se refiere a ellos como a peruanos iguales a todos los demás" (Mariátegui 1971:106), es la persistencia del privilegio del poder de casta, ahora mercantil, y del establecimiento de un modo basado en la profunda desigualdad que "el pueblo no tiene derecho a la instrucción [de calidad]" (Mariátegui 1971:106).

La globalización y la sociedad del conocimiento profundizan la mercantilización de la universidad contemporánea. Buscan transformarla según las necesidades del mercado en una institución donde los conocimientos son bienes y valores, únicamente existen porque permiten generar ganancias. Proceso neoliberal de la universidad impulsado por los expertos internacionales del Organización Mundial del Comercio (OMS) y el Banco Mundial (BM) que tienen la capacidad de transformarlos en políticas públicas de la educación superior. De esa forma, a partir de las dos últimas décadas está en desarrollo la formulación de políticas universitarias que buscan convertir la educación superior en un bien de consumo, propiciado la universidad empresa, la racionalización y la rentabilidad de los servicios. De esta forma, el Estado diseña un sistema único de ciencia y tecnología, mediante el Consejo Nacional de Ciencia y Tecnología (CONCYTEC), con la finalidad de establecer mecanismos que permitan alinear la inserción de la educación superior en la sociedad global del conocimiento y controlar la expansión de universidades y multiplicación de docentes en el país.

Las políticas de ciencia y tecnología que desarrolla CONCYTEC en las universidades buscan medir la producción del conocimiento para el mercado, siguiendo las pautas de la "excelencia", la "calidad del servicio", el "desempeño de gestión" y otras formas del logro mercantil. Se trata de evaluaciones y mediciones de la eficacia institucional en rankings universitarios y puntajes de los docentes, indicadores para clasificar el sistema de la universidad pública y de la comunidad de profesores, con la intención de quebrantar el sistema de universidades públicas y establecer jerarquías muy diferenciadas para la inversión y en los ingresos de los docentes, creando un nivel muy reducido de universidades top con un amplio apoyo económico y una mayoría que languidece por la escasez de recursos. De la misma forma delimitan una capa reducida de profesores con ingresos adicionales por ser más productivos, diferenciados de la inmensa mayoría con magros salarios. Las evaluaciones y mediciones definen un sistema-mundo del conocimiento educativo, delimitando una polarización extrema entre las universidades de las naciones centrales y las universidades periféricas y, al mismo tiempo, en el país reproducen un patrón universitario altamente jerarquizado que se ordena en función de las instituciones educativas elitizadas y condena a la mayoría a su inferiorización.

Proceso que genera una cultura administrativa y burocrática eurocéntrica, donde las universidades concentran presupuestos, destinan gran parte de recursos y desarrollan sus esfuerzos institucionales en la búsqueda a como dé lugar del licenciamiento y la acreditación permanente de las carreras y disciplinas según los moldes de las agencias globales, distrayendo la educación superior de las principales actividades de investigación y enseñanza. Propicia que los docentes se conviertan únicamente en hacedores de artículos en revistas indexadas, generalmente, elaboradas en el extranjero y bajo criterios eurocéntricos, por ejemplo, en Inglaterra el $97 \%$ de la revistas de impacto se encuentra en poder de un solo dueño: 
Taylor \& Francis (Santos 2007), alejando la posibilidad de elaborar libros orgánicos, muchos de ellos con más de 400 páginas, referidos a las necesidades del país y, sobre todo, relegando la estima de pensar lo propio y subvalorando la producción de conocimientos nacionales, que al ser evaluados con criterios universalizados poco o nada interesan a los grandes centros del saber europeos y norteamericanos. En el extremo, puede ocasionar que muchos de los profesores universitarios se transformen en simples llenadores de encuestas, informes de alumnos y demás materiales o exámenes de pruebas de comportamiento ético, al que destinan el 30\% del tiempo (Santos 2014) y, de esa forma, se devalúa completamente la labor de investigación y docencia en la educación superior.

La globalización neoliberal busca crear un sistema de producción de conocimiento universitario, controlado, disciplinado por las redes exteriores y validando únicamente el saber destinado al mercado, alejándolo de las posibles formas libres y críticas que responden a las necesidades propias del país. Bajo la visión de los diseños y pautas eurocéntricos, la burocratización de la vida universitaria peruana arrastraría su decadencia espiritual y epistémica.

Empero, pareciera que lo más saltante de las políticas globales que impulsa CONCYTEC es la integración de todas las áreas del conocimiento universitario en un único sistema de ciencia y tecnología, bajo el dominio de las disciplinas tecnológicas. Situación que introduce una tardía ruptura epistemológica de las "dos culturas" del conocimiento, la de las ciencias naturales que aparentemente se dirigen a la indagación de la verdad objetiva y, de otra parte, las humanidades y ciencias sociales, centradas en la búsqueda principalmente de la belleza y la ética. Esta separación sustancial en la educación universitaria no puede sino generar devastación de la heterogeneidad epistémica y degeneración de la democracia de los saberes. Se pretende tasar el conocimiento que producen las humanidades a partir de una concepción tomada de las llamadas ciencias básicas y de las tecnologías, propiciando una forma de "violencia epistémica" que ofende y desconoce el quehacer de los otros campos del saber y de los docentes.

Obligar a que la universidad se rija por criterios de las "dos culturas" es un acto de marginación epistémica que contradice el derecho democrático a la pluralidad de saberes. La universidad debe continuar siendo el espacio del debate de las diferencias entre las distintas formas de conocimiento. En el mundo actual, la ciencia conlleva una nueva alianza que permita integrar "el devenir cultural y natural" (Prigogine y Stengers 1983:325) y la unidad diferenciada de todos los conocimientos. En ese sentido, los conocimientos de todas las áreas deben ser valorados en sus propios términos, a partir de sus mismos criterios y procesos, y no a partir de una instancia exterior a ellos que se postula como garante de la verdad defendida por el Estado.

En general, el proceso de la universidad peruana arrastra un conjunto de permanencias que definen su carácter fundado en la colonialidad del saber. Proceso que tienen dos aspectos diferenciados y estrechamente interrelacionados. Uno se refiere al carácter eurocéntrico desde la fundación de la Universidad de San Marcos hasta la creación de un sistema único de ciencia y tecnología de las "dos culturas" y, lo otro, hace mención a la herencia del privilegio oligárquico, basado en un primer momento en la distinción de "sangre limpia" y, en tiempos recientes, en la distinción de la riqueza mercantil.

\section{La universidad y la revolución tecnológica de la información y la comunicación}

La universidad ha devenido en un campo de mutación fundamental en la sociedad contemporánea. La educación superior se encuentra en un momento de revisión profunda que incluyen los propios fundamentos del proceso de enseñanza, del modelo que permitió desarrollar la educación moderna desde el siglo XVI. 
El desarrollo de la revolución tecnológica de la información y la comunicación en el campo de la educación superior impulsa nuevas relaciones educativas, abre un campo de nuevas posibilidades de enseñanzaaprendizaje que anuncia otra universidad. Las nuevas tecnologías entran en conflicto con los viejos modelos educativos de la universidad. Las tecnología de la información y comunicación son el conjunto de procedimientos aplicados en el campo de la microelectrónica, la informática, las telecomunicaciones, teléfonos inteligentes, la digitalización y las redes que alteran las relaciones de enseñanza, facilitando los canales de comunicación y, sobre todo, concentran prácticamente toda la inmensidad del conocimiento en tiempo real. Actualmente el $95 \%$ de la información del mundo se encuentra digitalizada y la mayor parte es accesible en internet y otras formas informáticas.

La educación y la universidad se constituyeron en función de un "modelo tradicional" pedagógico que se desarrolló desde el siglo XVI hasta la actualidad, signado por la expansión de la modernización peruana. Es un patrón de aprendizaje pasivo, receptivo y memorístico, de simple transmisión de conocimientos y valores de las generaciones precedentes tomadas como verdades absolutas. Es un esquema de enseñanza instructivista: el profesor es el elemento fundamental que define la entrega de conocimientos, monopoliza el saber y tiene el papel activo en la exposición de las ideas. El aprendizaje se presenta como relaciones verticales de poder, en la que predomina la autoridad y la disciplina impuesta por el docente, de arriba hacia abajo, donde se encuentran los alumnos. Impone el conocimiento en la clase tipo conferencia, los apuntes, la memorización y los libros de texto. El alumno no desempeña una función importante, su labor es simplemente receptiva y es tratado como un mero objeto del aprendizaje.

La enseñanza se reduce exclusivamente al logos, el conocimiento se imparte únicamente en las aulas, bibliotecas y laboratorios, en un contexto rígido, cerrado y, en un ambiente puramente cognoscitivo, el alumno tiene que estar concentrado en escuchar al docente, no tiene la posibilidad de interactuar con sus compañeros y menos desarrollar otras actividades, de lo contrario puede ser sancionado, tiene que estar inmóvil y solo percibiendo al docente. Supone un proceso de enseñanza panóptico de control del cuerpo y mente. Por otra parte, es un modelo de transmisión del conocimiento monopolizado principalmente por la institución de la universidad. Toda la dinámica de la enseñanza se lleva exclusivamente en su campus, los conocimientos y valores hegemónicos se encuentran centralizados, enmarcados y diseñados por las instituciones educativas superiores.

En cambio, la nueva revolución tecnológica de la información y comunicación posibilita un proceso de enseñanza radicalmente diferente al modelo tradicional. La inmensa mayoría de los conocimientos y la información del mundo se encuentran disponibles en los dispositivos digitales, redes sociales y canales informáticos que hacen que todos los jóvenes y maestros tengan las mismas posibilidades de acceder y disponer de ellos. El aprendizaje-enseñanza se vuelve un proceso complejo, interactivo y participativo. En ese sentido, el modelo educativo que introduce las tecnologías de información y comunicación se basa en el constructivismo, perspectiva que concibe la enseñanza como una actividad autogenerada, crítica y compartida por los estudiantes. Las informaciones y discursos cognoscitivos existen en las redes y espacios tecnológicos que facilitan el acceso de los conocimientos a todos los que deseen aprender. Cada persona puede construir y organizar sus saberes según sus necesidades. Los jóvenes tienen la posibilidad de leer e interactuar mucho porque pueden acceder a varios espacios de información digitales y de redes que cambian constantemente. En ese marco, es algo muy censurable que la mayoría de las instituciones universitarias sigan educando según el modelo tradicional, sin haber entrado en las potencialidades de aprendizaje que introducen el mundo digital y la transformación de la comunicación. 
Las tecnologías de la información y comunicación posibilitan que la enseñanza no sea una simple transmisión de conocimientos. Aventuran por una acción horizontal pedagógica democrática, que concibe el aprendizaje como resultado de un proceso de construcción personal-colectiva de nuevos saberes, actitudes y experiencias, a partir de los ya existentes y en cooperación con los compañeros. Los nuevos medios de la información permiten que el aprendizaje incluya una dialógica integrada del logos y el mito, se aprende y se juega, se sueña y se aprende, se estudia y se realiza toda clase de actividades. El sujeto aprende en relación con los otros, en internet, círculos y hasta en movimientos sociales en redes. La enseñanza es parte de la vida social, ya no constituye un nivel apartado y especializado. Se trata de un aprendizaje que desborda la propia institución universitaria, los jóvenes pueden alcanzar la información y los conocimientos existentes en todo el mundo de internet, desde redes sociales, seminarios virtuales y múltiples plataformas digitales. Pero lo más significativo es que gran parte del aprendizaje puede ocurrir más allá de la oferta y demanda del mercado: se acerca al costo marginal cero. Los ejemplos mayores son la infinitud de páginas libres y Wikipedia, la mayor enciclopedia mundial. Los precios son casi inexistentes y no se cobra por participar, consultar y aprender, más bien predomina relaciones educativas de cooperación y en redes sociales.

El dominante patrón de colonialidad en la enseñanza-aprendizaje universitaria instructivista, vertical, cientificista e institucional, impide, obstaculiza y somete al despliegue del nuevo modelo educativo constructivista, horizontal, logos-mito y en redes que anuncian las tecnologías de la información y de la comunicación como otra posibilidad de la educación superior.

Si bien es cierto que las nuevas tecnologías establecen nuevas formas educativas, en el sistema universitario peruano no basta señalar los conflictos derivados del nuevo modelo pedagógico que introducen las novísimas tecnologías educativas. El problema principal son las capacidades, habilidades y destrezas de las personas y la sociedad para actuar y aprender a través de Internet, aspectos que dependen fundamentalmente del nivel educativo apropiado y cultural. De la forma como el sistema educativo prepare a los jóvenes para empoderarlos, para que puedan "aprender a aprender" por su propia cuenta, el problema se centra en la capacidad educativa disponible para moverse y estudiar en la galaxia de internet. En la educación superior lo esencial no es trasmitir información, sino generar la capacidad cognitiva de saberlos combinar en forma creativa, innovadora y adaptable a los cambios organizativos y tecnológicos del mundo actual. Aquí se encuentra el nudo central de la revolución tecnológica en la educación. El patrón educativo peruano es de los más eurocéntricos y dependientes, no solo porque se funda en la repetición de saberes y conocimientos elaborados íntegramente en los países desarrollados y copiados sin un tamiz de crítica adaptativa, sino que los conocimientos tienen un atraso escandaloso, en muchos casos del siglo XVIII o, en los casos extremos, simplemente son experiencias directas de estafa educativa, en la que no se trasmite ningún conocimiento, transformándose en nuevos mecanismos de colonialidad, de dominación cultural de la juventud peruana.

En esas condiciones, no basta que las universidades del país dispongan de Internet y nuevas tecnologías, sin un sistema de educación apropiado y con niveles muy bajos pueden llevar a que la juventud se pierda en el inmenso mundo virtual de información y no tenga capacidad para obtener los conocimientos requeridos para transformarlos en proyectos personales de desarrollo. En un sistema educativo masificado, mercantilizado y de muy bajos niveles de calidad, las nuevas tecnologías de aprendizaje se cosifican, se transfiguran en un discurso retórico y vacío de contenido, pierden su sentido histórico y devienen solo en meras prácticas lúdicas y efectistas de la información casi ilimitadas por los grupos de pares, propias de la sociedad del espectáculo. 
En este momento de grandes cambios en la vida universitaria que anuncian las tecnologías de información y comunicación, la colonialidad del saber da cuenta del conflicto agudo entre el nuevo modelo de enseñanza que indican las nuevas tecnologías y la persistencia hegemónica de las formas tradicionales, autoritarias, de la transmisión de conocimientos en la universidad peruana. Las nuevas tecnologías en la educación superior instan originales relaciones de enseñanza-aprendizaje, cuyo potencial de revolucionar la universidad contemporánea se paraliza por la existencia dominante del patrón educativo tradicional.

\section{Heterogeneidad de saberes en la universidad}

El nudo entre modernidad y conocimiento destaca por la particularidad de los profundos cambios históricos que vienen produciéndose en la producción epistémica en el siglo XXI. Asistimos a un proceso de propagación de una diversidad de formas de conocimientos en todos los ámbitos de la existencia. En América Latina se ensayan propuestas innovadoras y se muestra como una región de desarrollo muy fructífera, que buscan abrir el conocimiento a nuevas posibilidades.

De esa forma, el conocimiento es más que la simple comprensión universitaria. Entender la totalidad heterogénea de la existencia comporta sobrepasar el saber hegemónico eurocéntrico, implica arribar al reconocimiento de una unidad múltiple del mundo, inagotable en el tiempo y espacio, de espiritualidad y materialidad, de orden y caos, organización y trastorno, de lo humano y la naturaleza. Asimismo, el conocimiento universitario no puede reducirse únicamente a las limitaciones del positivismo y la racionalidad instrumental. Hacerlo dejaría de lado un vasto sector de la existencia social, mientras que la diversidad del mundo es infinita, lo que demanda la necesidad de un pensamiento complejo que subraye la dialógica de razón e imaginación, certeza y casualidad, sociedad e individuo, saber y emoción. La historia de América Latina se revela como un proceso muy heterogéneo que no solo posibilita la marcha de la racionalidad moderna sino, al mismo tiempo, incluye la manifestación de la ilusión, del individuo y la fuerza de la cultura milenaria, convirtiéndose en un camino sinuoso, lleno de bifurcaciones entre el logos y el mito.

En ese sentido, la producción del conocimiento del mundo heterogéneo implica una organización compleja que asume diversas formas relacionadas. Por un lado, en la universidad se destaca el encuentro conflictivo del saber corporativo hegemónico y de formas iniciales de saberes críticos. La producción del conocimiento corporativo imprime con su carácter a la universidad y la domina, configurado principalmente en los centros del saber de los países industrializados de Europa y Estados Unidos, que responde a las necesidades de la expansión de la mercantilización y de la expoliación de los recursos naturales del país. Por otro lado, dentro de los límites de los espacios democráticos de la universidad, se viene esbozando un saber crítico y a veces en confrontación con el saber hegemónico, siguiendo las pistas de un pensamiento que cuestiona el orden establecido o algunos de sus aspectos fundamentales. En particular se impugna la expansión de la desigualdad social y las relaciones cosificadas de la sociedad con la naturaleza, que Aníbal Quijano lo denomina "monstruo histórico" y "monstruo climático", como las mayores amenazas de la modernidad global.

Por otro lado, se legitima un conocimiento vital que se desarrolla fuera del ámbito universitario, resultado de la práctica de cientos de años de los pueblos originarios del país y de la experiencia de los movimientos de la sociedad, que condensan la "savia de nuestras civilizaciones ancestrales" (Fals Borda 2003:82) del mundo andino y amazónico. En las últimas décadas se ensayan diversas respuestas en el Perú. Los movimientos de la sociedad en su defensa del territorio y la ecología del mundo bosquejan un imaginario de la naturaleza como ser vivo, no solo como fuente de toda forma de vida sino, principalmente, como 
sujeto de vida, que se autoproduce en un proceso histórico de interrelaciones entre lo químico, biológico y lo físico, ser vivo con derechos plenos, perspectiva que rescata la vieja idea de las culturas andinas de la pachamama como Madre Naturaleza. De la misma forma, trabajadores, mujeres, jóvenes, homosexuales, pacifistas, antiutilitarios, antiglobalización y otros, desarrollan un conjunto de saberes, demandas y medidas de lucha que apuntan al reconocimiento de los derechos, solidaridad, reciprocidad y, en general, a la construcción de una sociedad más igualitaria y justa, que evocan la idea del "sujeto vivo es el: si tú eres, yo soy" (Hinkelammert 2015:127) como aspecto central de la producción democrática de la existencia social.

La producción del conocimiento se encuentra en un proceso de metamorfosis que empieza a conformarse más allá de la universidad. Las necesidades de la experiencia epistémica desbordan la centralidad del saber eurocéntrico de la educación superior, son parte de un entramado muy complejo.

La universidad ha perdido la centralidad y el control del conocimiento sobre el mundo por la gran diversidad de saberes que se desarrollan dentro y fuera del recinto educativo. La universidad ha perdido legitimidad, reconocimiento y prestigio frente a las múltiples formas de pensamiento, se ha diluido la capacidad de reconocimiento de ser el único actor productor de conocimiento.

La universidad peruana es una institución esencialmente monocultural y de predominio del saber moderno corporativo que prescinde de la enorme heterogeneidad de los otros saberes del país. La universidad recrea un espacio de desigualdad y colonialidad de las diversas formas de pensamiento, en la que se privilegia los saberes eurocéntricos, se constriñe los saberes críticos y excluye los conocimientos andinos y de los pueblos sometidos.

\section{Pensar la universidad peruana}

En América Latina en el contexto de la expansión de la globalización emergen pensamientos discordantes sobre la universalidad eurocéntrica y abren un momento crucial de disputa epistémica. La región rompe con el monodiscurso eurocéntrico y se transforma en fuente del pensar, imaginar y producir conocimiento que involucra activamente a la propia universidad.

En ese sentido, la universidad del Perú contemporáneo define su carácter en el debate e intersección entre las distintas posibilidades de educación superior. Sobresalen los siguientes desarrollos: La universidad empresa, la universidad neodesarrollista y la descolonialidad de la universidad.

En primer lugar, se destaca la persistencia de la universidad empresa. El predominio de la universidad empresa se viene consolidando en el Perú desde 1996 por la dación del Decreto Legislativo 882 como parte de las políticas neoliberales impulsadas por el régimen fujimorista. La universidad considera que debe su existencia solo al mercado y a la producción de bienes y servicios. La educación superior se "factoriza" para transformarse en una institución corporativa, en una empresa capitalista que sirve solo al proceso de modernización mercantil. Quizás los mayores ejemplos sean la Universidad Peruana de Ciencias Aplicadas (UPC) y Universidad San Ignacio de Loyola, pero, en realidad, corresponde al sistema de la universidad peruana desarrollada bajo el impulso neoliberal que ha definido su contenido como instituciones educativas privadas.

Proceso de mercantilización que igualmente envuelve al conjunto de las universidades públicas, privatizando parte de los servicios, cobrando por ellos y vendiendo productos del conocimiento, 
diversificando sus operaciones mediante la generación de ingresos propios. Lo más crítico, hace que toda la gestión universitaria se transforma en una entidad de negocios: los planes de estudio, los diplomas, los estudios de posgrado, la formación y la evaluación de docentes y estudiantes. En las universidades públicas alrededor del $50 \%$ del presupuesto tiene su origen en los recursos propios, obtenidos del mercado educativo.

En términos nacionales la universidad empresa está en contra de toda intervención pública: la mejor política es la que no existe. Se parte de la idea ilusoria que el mercado es el mejor y único distribuidor de los recursos de la sociedad. Para la universidad empresa el mercado es el centro de la vida social, es el medio que posibilita una sociedad perfecta, determinada por la "mano invisible" que lleva a la autorregulación y la armonía general en la modernidad capitalista. Patrocinada por el Banco Mundial, la universidad corporativa entiende que la educación tiene por finalidad exclusiva la formación de capital humano, de expertos, de jóvenes con competencias para que puedan producir bienes, servicios y competir en el mercado.

La universidad empresa se organiza profundamente en términos disciplinarios. Se naturaliza el establecimiento de las unidades académicas en forma vertical y cerradas para dar lugar a la gestión del conocimiento, tanto teórica como práctica, en la enseñanza y en la investigación. La universidad corporativa es el dominio de las estructuras eurocéntricas del saber. Los otros saberes no existen, se impide el desarrollo de los conocimientos críticos y se rechaza en forma absoluta los saberes indígenas y de los pueblos del país. La universidad empresa en el Perú pretende recuperar la hegemonía total de la razón eurocéntrica y reimponer el dominio de un universalismo cognoscitivo, proceso únicamente posible por la pretensión de imponer un nuevo periodo de colonización del saber en los países bajo el dominio epistémico actual.

Proceso de mercantilización de la educación que genera el debilitamiento extremo de las instituciones de la sociedad. El resultado es el desarrollo exponencial de las desigualdades. Se organiza un sistema universitario extremadamente jerarquizado, con un sector de cuatro o cinco universidades que son la élite, posesionadas con la venta de servicios, con los mejores docentes muy bien remunerados y pensiones muy altas de los alumnos, mientras que la amplia mayoría corresponden a una educación superior masificada de muy baja calidad, con docentes contratados por horas e ínfimos sueldos y sin otros servicios que ofrecer más que los estrictamente de enseñanza de pregrado y, a veces, de posgrado, que rayan en el facilismo y la compra-venta grados académicos.

Desde el Perú y América Latina la perspectiva de universidad empresa plantea un camino universitario solo por medio del mercado y los privilegios mercantiles. Nunca suscribió las viejas promesas de la modernidad de una educación para el desarrollo de un pensamiento propio y fundada en los valores de libertad e igualdad social.

En segundo lugar, la universidad neodesarrollista del siglo XXI. Es el discurso que formula no solo la recuperación de la tradición de la universidad pública, sino fija sus posibilidades institucionales de una educación que contribuya a la expansión de la modernización capitalista, poniendo énfasis en la figura de la "Razón de Estado", crítica al neoliberalismo para subrayar las potencialidades de la economía y la redistribución social, que en el caso de las experiencias asiáticas han logrado un éxito importante. En el Perú la propuesta fue enarbolada, de alguna manera, por la "segunda reforma universitaria". En general, este modelo universitario es únicamente entendible dentro de los límites del neodesarrollismo peruano y latinoamericano, que acentúa las afinidades del Estado con el sector privado, siguiendo las pautas de 
productividad y rentabilidad del mercado neoliberal, que destaca el fomento de las economías extractivistas.

La organización interna y administrativa de la universidad debe acomodarse a la formación de ciudadanos para el desarrollo productivo y social del país. La universidad forma ciudadanos con derechos a la educación pública y las necesidades del Estado. La universidad neodesarrollista persigue la educación gratuita, financiada principalmente por las entidades gubernamentales y la generación de conocimientos como bienes públicos que contribuyan a la expansión de las capacidades productivas del Estado y favorezcan la distribución de ingresos. En un contexto de alianza entre el Estado, la universidad y el mercado que contribuyan al crecimiento y la expansión de la modernización capitalista.

La universidad neodesarrollista insiste principalmente en la estructuración del departamentalismo y el conocimiento disciplinar con la esperanza de elevar y mejorar de la calidad académica. La producción del conocimiento recae en la propia universidad bajo las necesidades del impulso del Estado y la hegemonía de los saberes europeos y norteamericanos. Las formas críticas de pensamiento son marginales. Aunque pueden ser muy importantes, tienen poca relevancia en el contexto de la organización universitaria, y la presencia de los saberes indígenas y populares, cuando existen, se remiten a una representación cosificada, sin memoria y carentes de contenido histórico, aparecen como un legado acabado del pasado.

En la dimensión del dirigismo, los gobiernos, y las sociedades en general, tienden a aumentar la presión por la evaluación y monitoreo en un sistema ciencia y tecnología sobre las universidades públicas, acentuando el uso de normas y regímenes diseñados para garantizar el flujo de información centralizado y testificar la calidad de las instituciones educativas y de los docentes. De esa forma, una política centralizada de control, evaluación y fiscalización universitaria es parte de las entidades del Estado y en particular de CONCYTEC.

En general, la perspectiva de universidad neodesarrollista busca desvincularse del diseño neoliberal privatista y empresarial, fomentando la intervención educativa estatal para contribuir a la modernización capitalista, aunque dentro las fronteras eurocentricas del saber y en función de las industrias extractivas latinoamericanas.

Finalmente, la descolonialidad de la universidad es el discurso más integral de crítica para enfrentar las amenazas globales del neoliberalismo. No se trata solamente de una recuperación de la tradición de la educación pública. Lo fundamental es la elaboración de una propuesta cultural de la esperanza de la igualdad, fundada en un ideal moral que facilite la crítica a las diversas formas existentes de universidad.

La descolonialidad de la universidad es una promesa ideal de la responsabilidad educativa y de la cultura de la esperanza de la igualdad, no como modelo, sino más bien como principio ético que ordene una dirección de futuro, aliente una oferta de sentido que incite a la experiencia individual y permita movilizar las fuerzas latentes de la comunidad de docentes y alumnos. Ideal que inspira la crítica a los males de la universidad realmente existente, es el fundamento de la desaprobación de los abusos del presente. Contrapone tajantemente la realidad existente con el ideal moral de universidad. En ese proceso laberíntico, el ideal puede contribuir a trazar la descolonialidad de la universidad como un camino de experimentación del progreso humano, nunca como un proyecto terminado.

La descolonialidad de la universidad apunta al desarrollo de una cultura de la esperanza colaborativa enraizada por la producción de la autonomía epistémica y societal. La universidad se imagina 
principalmente en torno a un entramado de redes digitales institucionales y personales, globales y locales de intercambios de informaciones y conocimientos, que posibiliten el acceso igualitario a la ingente diversidad de saberes mediante un aprendizaje colaborativo, crítico y adaptable a los cambios tecnológicos y organizativos, utilizando preferentemente las nuevas pedagogías de e-learning y $m$-learning que operan en internet. Lo central de la universidad no es trasmitir información, sino crear capacidad de innovación y adaptación permanente al cambio societal. Como casi toda la información se encuentra en internet, el conocimiento se funda en un proceso cooperativo en las redes y de la capacidad de combinar información de modo creativo y adecuado.

Un mundo profundamente intercomunicado conlleva la conversión de las personas en sujetos con autonomía propia, con aquellos que comparten ideas semejantes en las redes sociales. La autonomía del sujeto frente a las instituciones de la sociedad abre la capacidad del individuo de ser protagonista de su existencia y, a la vez, portador de proyectos cognoscitivos de ideales comunes, como la preocupación por el medio ambiente y la igualdad social. Redes sociales y sujetos del conocimiento impulsan la transformación de la cultura en una cultura del acceso común y solidaria que trasciende en el tiempo y el espacio, que pueden hacer de la educación universitaria parte de la promesa histórica de un horizonte de sentido libertario.

Precisamente, la descolonialidad de la universidad es la idea magistral inspirada en la formación de seres humanos, la comprensión de los conocimientos y cualificaciones solo como bienes comunes para ser compartidos por todos, fundados en el valor de la responsabilidad como seres iguales socialmente, de reconocimiento mutuo y de unidad con la naturaleza. El valor de los conocimientos como bienes comunes de la humanidad solo tiene sentido fuera del cálculo mercantil y del precio, en conflicto permanente con la rentabilidad y el mercado.

De esa forma, la superación de las estructuras eurocéntricas del saber implica el desarrollo de un pensamiento propio que posibilite "construir paradigmas endógenos enraizados en nuestras propias circunstancias que reflejen la compleja realidad que tenemos y vivimos" (Fals Borda 2003:89). El conocimiento de la realidad solo puede emerger del contexto andino, amazónico y urbano peruano y latinoamericano, que tome en cuenta todo el legado cognoscitivo universal. Una perspectiva que facilite pensar la sociedad como parte de la naturaleza, originada en la igualdad social y libertad entre las personas.

La descolonialidad de la universidad supone una nueva institucionalidad. La universidad tiene que funcionar en una nueva estructura académica basada en la epistemología de la complejidad, que facilite ir más allá de la vieja organización disciplinaria y de control, perspectiva que enfatiza la comprensión del conocimiento como una totalidad, conformada por elementos heterogéneos y, a la vez, íntimamente interrelacionados. Que contribuya al establecimiento de un orden educativo que se defina en términos de temáticas de investigación y enseñanza. Perspectiva compleja que lleva a superar el saber eurocéntrico basado en los límites establecidos por la separación de las "dos culturas" entre las ciencias y las humanidades, es una apuesta por lograr una perspectiva que haga posible el conocimiento como una experiencia humana, de saberes integrados en una unidad heterogénea que sean, a la vez, objetivos, buenos y bellos. En este sentido, se trata del reconocimiento histórico del reencantamiento del conocimiento y el derecho democrático epistémico a la singularidad y a la diversidad de todos los saberes.

La descolonialidad de la universidad conlleva la crítica radical al dominio de discurso eurocéntrico para buscar transformarla en una institución de encuentro de igualdad intercultural de los conocimientos 
universitarios y los otros saberes andinos y populares que habían sido sometidos o excluidos. La "ecología de saberes" entre el saber científico y humanístico que la universidad produce y los "saberes legos, populares, tradicionales, urbanos, campesinos, provincianos" e indígenas (Santos 2007:67). El diálogo intercultural posibilitará que los otros saberes recuperen su memoria e identidad histórica que la universidad eurocéntrica siempre negó. La universidad fundada en la comprensión del logos y el mito posibilitan un mayor acercamiento a la infinita diversidad y complejidad del mundo.

La descolonialidad de la universidad es una propuesta que traza un camino más allá de la educación superior en la modernidad global. Otra universidad que alumbre la gestación de un nuevo horizonte de sentido donde la sociedad pueda pensarse como parte de la naturaleza y, a la vez, en igualdad social y libertad de las personas.

\section{Conclusiones}

Modernidad y educación universitaria en el Perú se conectan directamente con el proceso de universalización del saber. Desde su fundación, la universidad involucra el desarrollo de una visión del mundo moderno y de colonialidad, encarna una manera de pensar y una forma de producir conocimiento. En la universidad se difunde un discurso monocultural fundado en la episteme universal europeísta.

El proceso de la educación superior en el Perú que define su carácter eurocéntrico es resultado de las relaciones de la universidad y el poder establecido históricamente: Iglesia, Estado y Mercado. La universidad se ordena en función de los saberes teológicos de la conquista de las almas, la "Razón de Estado" y la educación por competencias para el mercado global. El eurocentrismo es el imaginario social, la memoria histórica y la perspectiva de conocimiento que permite organizar todas las subjetividades en un gran discurso universal que tienen su centro en Europa y los Estados Unidos.

El proceso de la universidad peruana es, a la vez, manifestación del orden de la colonialidad que impone el viejo privilegio educativo de casta, sea aristocrático de la limpieza de sangre y ahora de la riqueza mercantil, que considera que las mayorías poblacionales no tienen derechos educativos, menos los saberes indígenas y populares, por la exclusión directa de la universidad o de la educación de calidad.

La universidad peruana se organiza profundamente en términos disciplinares y en unidades académicas verticales y cerradas que clasifican el conocimiento en función de la hegemonía de algunas orientaciones teóricas, la teología en los primeros momentos, pasando por la formación de doctores y letrados y en el presente sobrevalorando las competencias técnicas, para desconocer y desvirtuar la democracia de la diversidad y singularidad de los saberes en la educación superior.

En el Perú contemporáneo el mercantilismo signa la pauta general del patrón universitario como parte del proceso de globalización neoliberal que cubre la sociedad. No obstante lo anterior, la educación superior es resultado de un proceso de transacción de las varias trayectorias de universidad que la definen y señalan un momento crucial de disputa sobre la organización de la producción cognoscitiva. Sobresale la presencia hegemónica de la universidad empresa, la universidad neodesarrollista y, desde los márgenes alumbra, la descolonialidad de la universidad.

A pesar de todo, pareciera que estaríamos asistiendo al desarrollo de una episteme que considera la posibilidad de existencia educativa más allá del mercado y de la presencia de solo el Estado, que permita 
pensar otra universidad en función de la comunidad de las redes y del acceso igualitario a los saberes, fundado en una cultura de la libertad y la esperanza.

\section{Bibliografía}

Burga, M. 2013. Universidad y poder. San Marcos y el Estado, pp. 305-315. En: M. Guisti y R. SáchezConcha. Universidad y nación. Lima: PUCP.

Castro-Gómez, S. 2014. Descolonizar las artes. Una genealogía del modelo de la universidad-empresa en Colombia. Bogotá: Ediciones de la Universidad Distrital Francisco José de Caldas.

Fals Borda, O. 2003. Ante la crisis del país. Ideas-acción para el cambio. Bogotá: El Áncora Editores.

Hinkelammert, F. 2015. Solidaridad o suicidio colectivo. San José: Arlekín.

Loayza, A. 2006. La política educativa del Estado peruano y las propuestas educativas de los colegios San Carlos y Nuestra Señora de Guadalupe: Lima, 1820-1857. Lima: UNMSM.

Lynch, N. 2016, 13 septiembre. Despojo a la universidad pública. La República. http://larepublica.pe/politica/972075-despojo-a-la-universidad-publica

Mariátegui, J.C. 1971. 7 ensayos de interpretación de la realidad peruana. Lima: Amauta.

Montoya, L. 2009. Desarrollo universitario y modernización en el Perú, pp. 135-159. En: J. Ríos. La universidad en el Perú: historia presente y futuro. Lima: ANR.

Prigogine, I. y Stengers, I. 1983. La nueva alianza. Metamorfosis de la ciencia. Madrid: Alianza.

Ribeiro, D. 2010. La universidad peruana, pp. 263-311. En: J. Ríos. La universidad en el Perú: historia presente y futuro. Lima: ANR.

Santos, B. de S. 2007. La universidad en el siglo XXI. Para una reforma democrática y emancipatoria de la universidad. La Paz: CIDES-UMSA.

Santos, B. de S. 2014. Universidad pública en el siglo XXI. Video YouTube

https://www.youtube.com/watch?v=M4jHoFw9C6g

Recibido el 14 Ago 2017

Aceptado el 25 Nov 2017 